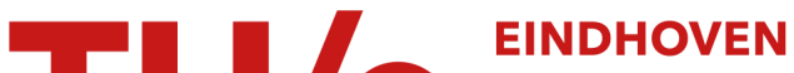 UNIVERSITY OF TECHNOLOGY
}

\section{Tail asymptotics of a Markov-modulated infinite-server queue}

Citation for published version (APA):

Blom, J. G., De Turck, K. E. E. S., Kella, O., \& Mandjes, M. R. H. (2014). Tail asymptotics of a Markovmodulated infinite-server queue. Queueing Systems: Theory and Applications, 78(4), 337-357.

https://doi.org/10.1007/s11134-014-9412-z

DOI:

10.1007/s11134-014-9412-z

Document status and date:

Published: 01/01/2014

Document Version:

Publisher's PDF, also known as Version of Record (includes final page, issue and volume numbers)

Please check the document version of this publication:

- A submitted manuscript is the version of the article upon submission and before peer-review. There can be important differences between the submitted version and the official published version of record. People interested in the research are advised to contact the author for the final version of the publication, or visit the $\mathrm{DOI}$ to the publisher's website.

- The final author version and the galley proof are versions of the publication after peer review.

- The final published version features the final layout of the paper including the volume, issue and page numbers.

Link to publication

\section{General rights}

Copyright and moral rights for the publications made accessible in the public portal are retained by the authors and/or other copyright owners and it is a condition of accessing publications that users recognise and abide by the legal requirements associated with these rights.

- Users may download and print one copy of any publication from the public portal for the purpose of private study or research.

- You may not further distribute the material or use it for any profit-making activity or commercial gain

- You may freely distribute the URL identifying the publication in the public portal.

If the publication is distributed under the terms of Article $25 \mathrm{fa}$ of the Dutch Copyright Act, indicated by the "Taverne" license above, please follow below link for the End User Agreement:

www.tue.nl/taverne

Take down policy

If you believe that this document breaches copyright please contact us at:

openaccess@tue.nl

providing details and we will investigate your claim. 


\title{
Tail asymptotics of a Markov-modulated infinite-server queue
}

\author{
J. Blom · K. De Turck · O. Kella · M. Mandjes
}

Received: 6 December 2013 / Revised: 3 May 2014 / Published online: 12 June 2014

(C) Springer Science+Business Media New York 2014

\begin{abstract}
This paper analyzes large deviation probabilities related to the number of customers in a Markov-modulated infinite-server queue, with state-dependent arrival and service rates. Two specific scalings are studied: in the first, just the arrival rates are linearly scaled by $N$ (for large $N$ ), whereas in the second in addition the Markovian background process is sped up by a factor $N^{1+\varepsilon}$, for some $\varepsilon>0$. In both regimes (transient and stationary) tail probabilities decay essentially exponentially, where the
\end{abstract}

Work done while K. de Turck was visiting Korteweg-de Vries Institute for Mathematics, University of Amsterdam, the Netherlands, with greatly appreciated financial support of Fonds Wetenschappelijk Onderzoek/Research Foundation-Flanders. He is also a Postdoctoral Fellow of the same foundation.

J. Blom $(\varangle) \cdot$ M. Mandjes

CWI, P.O. Box 94079, 1090 GB Amsterdam, The Netherlands

e-mail: joke.blom@cwi.nl

K. De Turck

Telin, Ghent University, St.-Pietersnieuwstraat 41, B9000 Gent, Belgium

e-mail: kdeturck@telin.ugent.be

O. Kella

Department of Statistics, The Hebrew University of Jerusalem, Jerusalem 91905, Israel

e-mail: Offer.Kella@huji.ac.il

M. Mandjes

Korteweg-de Vries Institute for Mathematics, University of Amsterdam, Science Park 904, 1098 XH Amsterdam, The Netherlands

e-mail: M.R.H.Mandjes@uva.nl

M. Mandjes

Eurandom, Eindhoven University of Technology, Eindhoven, The Netherlands

M. Mandjes

Ibis, Faculty of Economics and Business, University of Amsterdam, Amsterdam, The Netherlands 
associated decay rate corresponds to that of the probability that the sample mean of i.i.d. Poisson random variables attains an atypical value.

Keywords Queues · Infinite-server systems · Markov modulation · Large deviations

\section{Mathematics Subject Classification $\quad 60 \mathrm{~K} 25$}

\section{Introduction}

In $[2,3]$ we have considered the tail asymptotics of the Markov-modulated infiniteserver queue: under two specific scalings, large-deviations results were derived for the probability that the number of jobs in the system attains a given (atypical) value. In these queueing systems, both arrival and service processes depend on the state of an external, independently evolving finite-state Markov chain, referred to as the modulating process; the feature that there are infinitely many servers entails that customers are served in parallel.

As remarked in [5], however, such Markov-modulated infinite-server queues come in two flavors: one in which the service times are sampled upon arrival, and one in which the departure rate at a given point in time depends on the current state of the modulating process. Importantly, the large-deviations results in [2,3] relate to the former model; for the latter model such large-deviations asymptotics have not been derived so far, to the best of our knowledge. The primary objective of this paper is to identify these asymptotics.

The model considered in this paper can be specified in greater detail as follows. Let $J(\cdot)$ be an irreducible continuous-time Markov chain, on a finite-state space $\{1, \ldots, d\}$, with transition rate matrix $Q$ and stationary distribution vector $\pi$ (where we follow the convention that vectors are written in bold). When this modulating process, sometimes called the background process, is in state $i$, jobs arrive according to a Poisson process with rate $\lambda_{i} \geq 0$. In the context of our previous work [2,3], the service times were sampled upon arrival: if the state of $J(\cdot)$ is $i$ when the job arrives, then the service time is sampled from an exponential distribution with mean $1 / \mu_{i}$ (where it is noticed that the results could be extended to a setting with general state-dependent distributions). In the present paper, however, we consider the model in which the hazard rate of leaving the system at a given point in time, say $t$, is $\mu_{i}$ if $J(t)=i$. In our model there are infinitely many servers: there is no waiting. We denote by $M(t)$ the number of customers in the system at time $t \geq 0$; we assume the system being empty at time 0 .

The difference between the two models is reflected in a very insightful manner as follows. It was observed in [6] that in the model of [2,3] the number of customers in the system at time $t$ has, conditioned on the path $J(\cdot)$, a Poisson distribution with parameter

$$
\int_{0}^{t} \lambda_{J(s)} e^{-\mu_{J(s)}(t-s)} \mathrm{d} s .
$$

This property can be intuitively understood by realizing that $e^{-\mu_{i}(t-s)}$ can be interpreted as the probability that a customer arriving at time $s \in[0, t)$ while the back- 
ground process is in state $i$, is still present at time $t$. For the model to be considered in the present paper, a similar representation is valid: $M(t)$ has again a Poisson distribution, but now with random parameter

$$
\int_{0}^{t} \lambda_{J(s)} e^{-\int_{s}^{t} \mu_{J(r)} \mathrm{d} r} \mathrm{~d} s .
$$

Observe how the state-dependent departure rate is incorporated in this expression: now $\exp \left(-\int_{s}^{t} \mu_{J(r)} \mathrm{d} r\right)$ represents the probability that a customer arriving at time $s \in[0, t)$ is still present at time $t$. As it will turn out, the representation (2) will enable us to derive the large deviations asymptotics that we are aiming for.

The literature on Markov-modulated infinite-server queues is surprisingly small (compared to the literature on Markov-modulated single-server queues); we mention a number of key papers here. O'Cinneide and Purdue [12] provide explicit expressions for the moments of the stationary number of customers, and systems of partial differential equations for the corresponding transient moments, in the context of the model variant studied in the present paper (with state-dependent hazard rate, that is). Related results, for a considerably broader class of models, are given in [10]; we also mention [9] for extensions to a semi-Markovian background process. As mentioned above, [6] presents the useful observation that $M(t)$ has a Poisson law with a random parameter (that depends on the path of the background process in $[0, t]$ ), as was highlighted in (1) and (2).

In $[1,4]$ the arrival rates are scaled by $N$ (to become $N \lambda_{i}$ when $J(\cdot)$ is in state $i$ ), while the background process is sped up by a factor $N^{\alpha}$. For both model variants introduced above, central-limit type results are derived. A crucial finding is that results in which the background process is faster than the arrival process $(\alpha>1$, that is) are intrinsically different from those in which the background process is slower $(\alpha<1)$. A similar dichotomy applies in the large-deviations domain for the model in which the service times are sampled upon arrival, corresponding to representation (1); [2] covers the case of a slowly moving background process and [3] the case of a fast background process. The results presented in the present paper show that these qualitative findings carry over to the model variant in which the departure rates depend on the current state of the background process, that is, the variant corresponding to representation (2).

The organization and contributions of this paper are as follows.

- In Sect. 2 we consider the counterpart of [2]: we study the regime in which only the arrival rates are scaled by a factor $N$, for $N$ large. It turns out that, with $M^{(N)}(t)$ the number of customers in the system in the $N$-scaled model, the tail probabilities of $M^{(N)}(t)$ decay exponentially, where the corresponding decay rate is the solution of a specific optimization problem. This optimization problem lends itself to a nontrivial explicit solution, in terms of a closed-form expression for the most likely path followed by the background process in order for the number of customers to reach a high value. Given this explicit result, the large-deviations asymptotics follow from a proof that resembles the one in [2].

- Section 3 addresses the counterpart of [3]: we scale the arrival rates by $N$, but the transition rates of the background process by $N^{1+\varepsilon}$ for $\varepsilon>0$. Defining 


$$
\lambda_{\infty}:=\sum_{i=1}^{d} \pi_{i} \lambda_{i}, \quad \mu_{\infty}:=\sum_{i=1}^{d} \pi_{i} \mu_{i}
$$

the main intuition is that in this scaling, as $N$ tends to $\infty$, the arrival process becomes essentially Poisson (with rate $N \lambda_{\infty}$ ), while the service times become exponentially distributed with a uniform service rate (with mean $\mu_{\infty}^{-1}$ ), so that the system behaves as an $\mathrm{M} / \mathrm{M} / \infty$ queue with these parameters. This explains why the large deviations of the (transient and stationary) number of customers in the system are those of the sample mean of i.i.d. Poisson random variables.

As an aside, we mention that the large deviations for the case of $\varepsilon=0$ have been studied in [8], focusing on the case that $d=2$.

\section{Slow timescale regime}

In this section, we consider the regime in which the arrival rates $\lambda_{i}$, for $i=1, \ldots, d$ are scaled by $N$, whereas the generator matrix $Q$ remains unchanged. In Sect. 2.1 we prove a number of structural properties related to the maximum (and minimum) value that can be attained by the random parameter of the Poisson distribution, cf. representation (2). These results are then used in Sect. 2.2 when establishing large deviations results.

\subsection{Maximum value attained by Poisson parameter}

The objective of this section is to find a path $f^{+}(\cdot)$ for $J(s), 0 \leq s \leq t$ that maximizes the (random) parameter of the Poisson distribution (2). Let $\mathscr{G}_{t}$ denote the class of Borel functions $f:[0, t] \mapsto\{1, \ldots, d\}$ and, for a given $f \in \mathscr{G}_{t}$, denote the Poisson parameter by:

$$
\kappa_{t}(f):=\int_{0}^{t} \lambda_{f(s)} e^{-\int_{s}^{t} \mu_{f(r)} \mathrm{d} r} \mathrm{~d} s .
$$

We thus want to solve the following optimization problem:

$$
\sup _{f \in \mathscr{G}_{t}} \kappa_{t}(f) \equiv \kappa_{t}^{+}
$$

and we seek a maximizing path $f^{+}$satisfying

$$
\kappa_{t}\left(f^{+}\right)=\kappa_{t}^{+} .
$$

As it turns out in Sect. 2.2, such a path, which will be shown to exist and is Lebesgue almost-surely unique, plays a crucial role when determining the large deviation asymptotics of the number of customers in the system in our $N$-scaled model. We will also point out how to identify a path $f^{-}(\cdot)$ that minimizes $\kappa_{t}(f)$. 
In preparation to analyzing the optimization problem (P), define $\varrho_{i}:=\lambda_{i} / \mu_{i}$. An important role is played by an index $i^{+}$(not necessarily unique) that satisfies $\varrho_{i^{+}}=\varrho^{+}:=\max _{i \in\{1, \ldots, d\}} \varrho_{i}$.

Lemma 1 The following claims hold:

1. For every $f \in \mathscr{G}_{t}$,

$$
\kappa_{t}(f) \leq \varrho^{+}\left(1-e^{-\int_{0}^{t} \mu_{f(r)} \mathrm{d} r}\right)<\varrho^{+}
$$

2. For any $t \geq 0$,

$$
\kappa_{t}^{+} \geq \varrho^{+}\left(1-e^{-\mu_{i}+t}\right)
$$

Proof Claim 1 is an immediate consequence of

$$
\begin{aligned}
\kappa_{t}(f) & =\int_{0}^{t} \varrho_{f(s)} \mu_{f(s)} e^{-\int_{s}^{t} \mu_{f(r)} \mathrm{d} r} \mathrm{~d} s \leq \varrho^{+} \int_{0}^{t} \mu_{f(s)} e^{-\int_{s}^{t} \mu_{f(r)} \mathrm{d} r} \mathrm{~d} s \\
& =\varrho^{+}\left(1-e^{-\int_{0}^{t} \mu_{f(r)} \mathrm{d} r}\right)
\end{aligned}
$$

whereas Claim 2 follows from considering the constant function $f(s)=i^{+}$for $s \in$ $[0, t]$, so that:

$$
\kappa_{t}^{+} \geq \int_{0}^{t} \lambda_{i}+e^{-\mu_{i+(t-s)}} \mathrm{d} s=\varrho^{+}\left(1-e^{-\mu_{i+} t}\right) .
$$

This proves the claims.

In the following, it will prove useful to represent the elements of the set of combinations of arrival rates and service rates, i.e., $\left\{\left(\mu_{i}, \lambda_{i}\right) \mid i \in\{1, \ldots, d\}\right\}$, as points in the $(\mu, \lambda)$ plane.

Lemma 2 Let $\varrho^{+}$be the maximum slope between the origin and any $\left(\mu_{i}, \lambda_{i}\right)$. There exists a finite sequence of different states $i_{1}, \ldots, i_{k}$ and values $0=I_{0}<\cdots<I_{k}=$ $\varrho^{+}$such that for every $0 \leq c<\varrho^{+}$we have

$$
\max _{i \in\{1, \ldots, d\}}\left(\lambda_{i}-c \mu_{i}\right)=\sum_{\ell=1}^{k}\left(\lambda_{i_{\ell}}-c \mu_{i_{\ell}}\right) 1_{\left[I_{\ell-1}, I_{\ell}\right)}(c) .
$$

Proof Algorithm 1 (see Appendix) is a constructive method to find a minimal nonnegative, nondecreasing, concave function $g$ such that that $g\left(\mu_{i}\right) \geq \lambda_{i}$ for all $i \in\{1, \ldots, d\}$. This function is unique (the infimum of concave functions is concave); $i_{1}, \ldots, i_{k}$ are the indices for which $g\left(\mu_{i}\right)=\lambda_{i}$, that is, the indices of the extreme points of the 
hypograph. $I_{0}$ is the slope zero, $I_{j}$ is the slope of the segment connecting $\left(\mu_{i_{j}}, \lambda_{i_{j}}\right)$ and $\left(\mu_{i_{j+1}}, \lambda_{i_{j+1}}\right)$.

For any $0<v<\infty$ there is some supergradient $c(v) \in\left[0, \varrho^{+}\right]$, such that for each $0 \leq u<\infty, g(u)-g(v) \leq c(v)(u-v)$, that is, $g(u)-c(v) u \leq g(v)-c(v) v$. In particular, if we take $v=\mu_{i_{\ell}}$, then $g(v)=\lambda_{i_{\ell}}$ and a supergradient is any $c \in\left[I_{\ell-1}, I_{\ell}\right]$ which gives for each $i$,

$$
\lambda_{i}-c \mu_{i} \leq g\left(\mu_{i}\right)-c \mu_{i} \leq g\left(\mu_{i_{\ell}}\right)-c \mu_{i_{\ell}}=\lambda_{i_{\ell}}-c \mu_{i_{\ell}} .
$$

This proves the stated lemma.

In an identical manner, for the purpose of minimization (rather than maximization), we need to find the maximal nondecreasing convex function $h$ such that $h\left(\mu_{i}\right) \leq$ $\lambda_{i}$ for each $i$ with maximal subgradient $\varrho^{-}:=\min _{i \in\{1, \ldots, d\}} \varrho_{i}$. In the maximizing case, the restriction on the maximal subgradient was automatically satisfied by the assumption that the function must be nonnegative and thus $g(0)=0$, which implies that the maximal supergradient is $\varrho^{+}$(the slope of the segment that connects $(0,0)$ with $\left.\left(\mu_{i_{k}}, \lambda_{i_{k}}\right)\right)$.

Lemma 3 The output of Algorithm 2 (see Appendix) is a sequence of different states $i_{1}, \ldots, i_{k}$ and values $0=I_{0}<\cdots<I_{k}=\varrho^{-}$such that for every $0 \leq c<\varrho^{-}$we have

$$
\min _{i \in\{1, \ldots, d\}}\left(\lambda_{i}-c \mu_{i}\right)=\sum_{\ell=1}^{k}\left(\lambda_{i_{\ell}}-c \mu_{i_{\ell}}\right) 1_{\left[I_{\ell-1}, I_{\ell}\right)}(c) .
$$

We are now ready to state the main result of this subsection, presenting the maximizing path explicitly; later we also point out how the corresponding minimizing path can be constructed. We first introduce some notation. Let the sequence $i_{1}, \ldots, i_{k}$ be as in Lemma 2 . Let the time epochs $t_{0}^{+}, \ldots, t_{k-1}^{+}$be defined recursively through $t_{0}^{+}:=0, t_{k}^{+}:=\infty$ and, for $\ell \in\{1, \ldots, k-1\}$,

$$
t_{\ell}^{+}:=t_{\ell-1}^{+}+\frac{1}{\mu_{i_{\ell}}} \log \frac{\varrho_{i_{\ell}}-I_{\ell-1}}{\varrho_{i_{\ell}}-I_{\ell}}
$$

Also, set $f^{+}(s)=i_{\ell}$ for $s \in\left[t_{\ell-1}^{+}, t_{\ell}^{+}\right)$and $\ell \in\{1, \ldots, k\}$.

Theorem $1 f^{+}$is optimal for $(\mathrm{P})$ for each $t \geq 0$. If $t_{\ell-1}^{+} \leq t<t_{\ell}^{+}$then the optimal value is given by

$$
\kappa_{t}^{+}=\kappa_{t}\left(f^{+}\right)=I_{\ell-1} e^{-\mu_{i_{\ell}}\left(t-t_{\ell-1}^{+}\right)}+\varrho_{i_{\ell}}\left(1-e^{-\mu_{i_{\ell}}\left(t-t_{\ell-1}^{+}\right)}\right) .
$$

Proof We observe that

$$
\kappa_{t}(f)=\int_{0}^{t}\left(\lambda_{f(s)}-\mu_{f(s)} \kappa_{s}(f)\right) \mathrm{d} s
$$


since the derivatives of the integral above and (4) are equal and $\kappa_{t}(0)=0$. Thus $(\mathrm{P})$ is equivalent to an optimal control problem $\left(\mathrm{P}^{\prime}\right)$ of the form

$$
\left\{\begin{aligned}
\operatorname{maximize} & x(t) \\
\text { subject to: } & x^{\prime}(s)=\lambda_{f(s)}-\mu_{f(s)} x(s), s \in[0, t] \\
& x(0)=0 \\
& f \in \mathscr{G}_{t} .
\end{aligned}\right.
$$

The Hamiltonian-as used in optimal control theory-for $\left(\mathrm{P}^{\prime}\right)$ is $H(x, p, f, s)=$ $p(s)\left(\lambda_{f(s)}-\mu_{f(s)} x(s)\right)$ where $p(s)=e^{-\int_{s}^{t} \mu_{f(r)} \mathrm{d} r}$ is the unique solution of

$$
p^{\prime}(s)=-\partial_{x} H(x, p, f)=\mu_{f(s)} p(s)
$$

and $p(t)=1$. As $p$ is positive, $f^{+}$maximizes $H(x, p, f)$ if and only if it maximizes $\lambda_{f}-\mu_{f} x$. As a consequence, the Pontryagin maximum principle hints at the guess that an optimal solution should satisfy for each $s \in[0, t]$ :

$$
\lambda_{f^{+}(s)}-\mu_{f^{+}(s)} \kappa_{s}^{+}=\max _{i \in\{1, \ldots, d\}}\left(\lambda_{i}-\mu_{i} \kappa_{s}^{+}\right)
$$

Assuming that this is the correct guess, and recalling that $i^{+}=\arg \max \varrho_{i}$, we have that

$$
\max _{i \in\{1, \ldots, d\}}\left(\lambda_{i}-\mu_{i} \kappa_{s}^{+}\right) \geq \lambda_{i^{+}}-\mu_{i}+\kappa_{s}^{+}=\mu_{i}+\left(\varrho^{+}-\kappa_{s}^{+}\right)>0
$$

and it follows by combining part 1 of Lemma 1 with Eq. (10) that $\kappa_{s}^{+}$is strictly increasing, continuous, with $\kappa_{0}^{+}=0$ and $\kappa_{s}^{+} \rightarrow \varrho^{+}$as $s \rightarrow \infty$.

By Lemma 2, for every $\ell \in\{1, \ldots, k\}$ and $s$ such that $I_{\ell-1} \leq \kappa_{s}^{+}<I_{\ell}$, we have that

$$
\arg \max _{i \in\{1, \ldots, d\}}\left(\lambda_{i}-\kappa_{s}^{+} \mu_{i}\right)=i_{\ell}
$$

and in order to describe the optimal control and the value of $\kappa_{t}^{+}$(the optimal value of $\kappa_{t}(f)$ ), for each $t \geq 0$, it remains to find $t_{0}^{+}<\cdots<t_{k}^{+}$such that $I_{\ell-1} \leq \kappa_{s}^{+}<I_{\ell}$ if and only if $t_{\ell-1}^{+} \leq s<t_{\ell}^{+}$. For this purpose it is straightforward to show that

$$
I_{\ell}=I_{\ell-1} e^{-\mu_{i_{\ell}}\left(t_{\ell}^{+}-t_{\ell-1}^{+}\right)}+\varrho_{i_{\ell}}\left(1-e^{-\mu_{i_{\ell}}\left(t_{\ell}^{+}-t_{\ell-1}^{+}\right)}\right)
$$

and some simple manipulations result in (8). In particular, for $\ell=k$ the equality in (11) can be achieved only with $t_{k}^{+}=\infty$. For $I_{\ell-1} \leq t<I_{\ell}$, replacing $I_{\ell}$ on the left by $\kappa_{t}^{+}$and $t_{\ell}^{+}$on the right by $t$, results in (9).

To complete the proof, we need to show that our guess is indeed the correct one. If not, then there would be some choice of $f \in \mathscr{G}_{t}$ such that $\kappa_{t}(f)>\kappa_{t}^{+}$. Note that both $\kappa_{t}^{+}$and $\kappa_{t}(f)$ are (absolutely) continuous functions of $t$, and satisfy $\kappa_{0}^{+}=\kappa_{0}(f)=0$. 
Now introduce $\tau_{t}(f):=\sup \left\{s: s \leq t, \kappa_{s}^{+}=\kappa_{s}(f)\right\}$. By continuity it follows that $\kappa_{\tau_{t}(f)}^{+}=\kappa_{\tau_{t}(f)}(f)$, with $\tau_{t}(f)<t$, and in addition that $\kappa_{s}^{+}<\kappa_{s}(f)$ for each $\tau_{t}(f)<s \leq t$. Hence, for any $\tau_{t}(f)<s \leq t$,

$$
\begin{aligned}
\lambda_{f(s)}-\mu_{f(s)} \kappa_{s}(f) & \leq \max _{i \in\{1, \ldots, d\}}\left(\lambda_{i}-\mu_{i} \kappa_{s}(f)\right)<\max _{i \in\{1, \ldots, d\}}\left(\lambda_{i}-\mu_{i} \kappa_{s}^{+}\right) \\
& =\lambda_{f^{+}(s)}-\mu_{f^{+}(s)} \kappa_{s}^{+}
\end{aligned}
$$

which implies that

$$
\begin{aligned}
\kappa_{t}(f)-\kappa_{\tau_{t}(f)}(f) & =\int_{\tau_{t}(f)}^{t}\left(\lambda_{f(s)}-\mu_{f(s)} \kappa_{s}(f)\right) \mathrm{d} s \\
& <\int_{\tau_{t}(f)}^{t}\left(\lambda_{f^{+}(s)}-\mu_{f^{+}(s)} \kappa_{s}^{+}\right) \mathrm{d} s=\kappa_{t}^{+}-\kappa_{\tau_{t}(f)}^{+},
\end{aligned}
$$

and since $\kappa_{\tau_{t}(f)}(f)=\kappa_{\tau_{t}(f)}^{+}$we have $\kappa_{t}(f)<\kappa_{t}^{+}$, a contradiction. Thus, $\kappa_{t}(f) \leq$ $\kappa_{t}^{+}=\kappa_{t}\left(f^{+}\right)$for every $f \in \mathscr{G}_{t}$ (and every $t \geq 0$ ), and conclude that $f^{+}$is indeed optimal.

The corresponding minimization (rather than maximization) problem can be dealt with analogously. In Theorem 1 we should now take the sequence $i_{1}, \ldots, i_{k}$ as in Lemma 3 (that is, the output of Algorithm 2). Let $f^{-}$be a minimizing path, which is, like $f^{+}$, Lebesgue almost-surely unique.

\subsection{Large-deviations results}

We have already noticed that $M^{(N)}(t)$ has a Poisson distribution with (random) parameter $N \kappa(J)$, with $J \equiv(J(s))_{s \in[0, t]}$ the path of the background process. Below we identify two numbers $a^{+}$and $a^{-}$such that for all $a<a^{+}\left(a>a^{-}\right)$the exponential decay rate of the above transient overflow (underflow) probability equals 0 ; the striking feature, however, is that $a^{+}$is strictly larger than $a^{-}$. To keep the notation transparent, we suppress the dependence on $t$ of functions and variables. Let $P^{(N)}(f)$ denote a Poisson random variable with mean $N \kappa(f)$, and let $\mathscr{G}_{t}$ be as defined before. Combining the above, we can write, in self-evident notation,

$$
\mathbb{P}\left(M^{(N)}(t)>N a\right)=\int_{f \in \mathscr{G}_{t}} \mathbb{P}\left(P^{(N)}(f)>N a\right) \mathbb{P}(J(\cdot) \in \mathrm{d} f(\cdot)) .
$$

Define

$$
d(f):=a-\kappa(f)-a \log \frac{a}{\kappa(f)}
$$


For $f^{+}$and $f^{-}$, the following lemma is an immediate consequence of Theorem 1 and its minimization counterpart.

Lemma 4 Both $f^{+}(\cdot)$ and $f^{-}(\cdot)$ are piecewise constant functions, taking values in $\{1, \ldots, d\}$, that jump at most $d-1$ times in $[0, t]$.

We now state and prove the main result of this subsection.

Theorem 2 For $a \geq a^{+}:=\kappa\left(f^{+}\right)$,

$$
\lim _{N \rightarrow \infty} \frac{1}{N} \log \mathbb{P}\left(M^{(N)}(t)>N a\right)=d\left(f^{+}\right)
$$

For $a \leq a^{-}:=\kappa\left(f^{-}\right)$,

$$
\lim _{N \rightarrow \infty} \frac{1}{N} \log \mathbb{P}\left(M^{(N)}(t)<N a\right)=d\left(f^{-}\right) .
$$

Proof Although the proof is similar to that of [2, Theorem 1], we include it here for the sake of completeness and readability. We focus in the proof on the case that $a \geq a^{+}$; the case $a \leq a^{-}$works analogously.

$\triangleright$ We start by proving the lower bound. Recall that the jump epochs in $[0, t]$ corresponding to $f^{+}$, resulting from Lemma 4, are denoted by $t_{1}^{+}, \ldots, t_{k}^{+}$, with $k<d$. Introduce the following set of functions that are 'close to' $f^{+}$(i.e., equal to $f^{+}$, apart from 'small' intervals around the $t_{j}^{+}, j=1, \ldots, k$ ):

$$
\mathscr{G}_{t, \delta}:=\left\{f \in \mathscr{G}_{t}: f(s)=f^{+}(s) \text { for all } s \in[0, t] \backslash \bigcup_{j=1}^{k}\left(t_{j}^{+}-\delta, t_{j}^{+}+\delta\right)\right\}
$$

choose $\delta>0$ sufficiently small that the intervals $\left(t_{j}^{+}-\delta, t_{j}^{+}+\delta\right)$ do not overlap nor cover times 0 and $t$. Consider the following obvious lower bound:

$$
\mathbb{P}\left(M^{(N)}(t)>N a\right) \geq\left(\min _{f \in \mathscr{G}_{t, \delta}} \mathbb{P}\left(P^{(N)}(f)>N a\right)\right) \mathbb{P}\left(J(\cdot) \in \mathscr{G}_{t, \delta}\right) .
$$

Now it is realized that $\mathbb{P}\left(J(\cdot) \in \mathscr{G}_{t, \delta}\right)$ is strictly positive (where it is used that $J(\cdot)$ is an irreducible Markov chain on a finite state space), and in addition independent of $N$. This entails that

$$
\liminf _{N \rightarrow \infty} \frac{1}{N} \log \mathbb{P}\left(M^{(N)}(t)>N a\right) \geq \liminf _{N \rightarrow \infty} \frac{1}{N} \log \left(\min _{f \in \mathscr{G}_{t, \delta}} \mathbb{P}\left(P^{(N)}(f)>N a\right)\right) .
$$


Then observe that, due to Stirling's factorial approximation, if $a \geq \kappa(f)$, for any $\varepsilon>0$ and $N$ large enough,

$$
\begin{aligned}
\mathbb{P}\left(P^{(N)}(f) \geq N a\right) & =\sum_{k \geq N a} e^{-N \kappa(f)} \frac{(N \kappa(f))^{k}}{k !} \\
& \geq e^{-N \kappa(f)} \frac{(N \kappa(f))^{\lceil N a\rceil}}{\lceil N a\rceil !} \geq e^{N d(f)} \frac{1-\varepsilon}{\sqrt{2 \pi N a}}
\end{aligned}
$$

Choose an arbitrary $f \in \mathscr{G}_{t, \delta}$. Then define $\lambda^{+}:=\max _{i} \lambda_{i}$, and $\mu^{+}:=\max _{i} \mu_{i}$. The triangle inequality implies that $\left|\kappa(f)-\kappa\left(f^{+}\right)\right|$is majorized by

$$
\begin{aligned}
& \left|\int_{0}^{t} \lambda_{f(s)} e^{-\int_{s}^{t} \mu_{f(r)} \mathrm{d} r} \mathrm{~d} s-\int_{0}^{t} \lambda_{f(s)} e^{-\int_{s}^{t} \mu_{f^{+}(r)} \mathrm{d} r} \mathrm{~d} s\right| \\
& +\left|\int_{0}^{t} \lambda_{f(s)} e^{-\int_{s}^{t} \mu_{f^{+}(r)} \mathrm{d} r} \mathrm{~d} s-\int_{0}^{t} \lambda_{f^{+}(s)} e^{-\int_{s}^{t} \mu_{f^{+}(r)} \mathrm{d} r} \mathrm{~d} s\right| .
\end{aligned}
$$

Due to the definition of the set $\mathscr{G}_{t, \delta}$, the latter of these two terms is majorized by

$$
\int_{0}^{t}\left|\lambda_{f(s)}-\lambda_{f^{+}(s)}\right| e^{-\int_{s}^{t} \mu_{f^{+}(r)} \mathrm{d} r} \mathrm{~d} s \leq \int_{0}^{t}\left|\lambda_{f(s)}-\lambda_{f^{+}(s)}\right| \mathrm{d} s \leq 2 \lambda^{+} \delta k .
$$

Now focus on the former term. First observe that, for all $s \in[0, t]$,

$$
\left|\int_{s}^{t}\left(\mu_{f(r)}-\mu_{f^{+}(r)}\right) \mathrm{d} r\right| \leq 2 \mu^{+} \delta k
$$

so that the term can be bounded by

$$
\begin{aligned}
& \int_{0}^{t} \lambda_{f(s)} e^{-\int_{s}^{t} \mu_{f(r)} \mathrm{d} r}\left|1-e^{-\int_{s}^{t}\left(\mu_{f^{+}(r)}-\mu_{f(r)}\right) \mathrm{d} r}\right| \mathrm{d} s \leq t \lambda^{+} \\
& \quad \times \max \left\{1-e^{-2 \mu^{+} \delta k}, e^{2 \mu^{+} \delta k}-1\right\} .
\end{aligned}
$$

We conclude that $\left|\kappa(f)-\kappa\left(f^{+}\right)\right|$goes to 0 as $\delta \downarrow 0$. As a consequence, also $\mid d(f)-$ $d\left(f^{+}\right) \mid$vanishes as $\delta \downarrow 0$. From this, we conclude that for $a \geq \kappa\left(f^{+}\right)$,

$$
\liminf _{N \rightarrow \infty} \frac{1}{N} \log \mathbb{P}\left(M^{(N)}(t)>N a\right) \geq d\left(f^{+}\right) .
$$


$\triangleright$ The corresponding upper bound is less involved; the proof is identical to that of [2, Theorem 1]. Note that if $a>a^{+}$, then for all $f \in \mathscr{G}_{t}$ we have $\mathbb{E} P^{(N)}(f)$ is smaller than or equal to $\mathrm{Na}$. Evidently,

$$
\mathbb{P}\left(M^{(N)}(t)>N a\right) \leq \max _{f \in \mathscr{G}_{t}} \mathbb{P}\left(P^{(N)}(f)>N a\right)
$$

Based on the Chernoff bound [7], we have

$$
\mathbb{P}\left(P^{(N)}(f)>N a\right) \leq e^{N d(f)}
$$

Combining the above inequalities, we obtain

$$
\limsup _{N \rightarrow \infty} \frac{1}{N} \log \mathbb{P}\left(M^{(N)}(t)>N a\right) \leq \max _{f \in \mathscr{G}_{t}} d(f) .
$$

As $\kappa\left(f^{+}\right)$maximizes $\kappa(f)$, and $d(f)$ is increasing in $\kappa(f)$ (for $\kappa(f) \leq a$ ), we conclude that

$$
\limsup _{N \rightarrow \infty} \frac{1}{N} \log \mathbb{P}\left(M^{(N)}(t)>N a\right) \leq d\left(f^{+}\right) .
$$

This proves the upper bound.

Recall that $\varrho^{-}:=\min _{i \in\{1, \ldots, d\}} \varrho_{i}$, where $i^{-}$is such that $\varrho_{i^{-}}=\varrho^{-}$. Then the following result, featuring the large deviations of the steady-state $M^{(N)}$ of $M^{(N)}(t)$, follows from a small modification in the proof of Theorem 2, in complete analogy to [2, Proposition 1].

Corollary 1 For $a \geq a^{+}:=\varrho^{+}$,

$$
\lim _{N \rightarrow \infty} \frac{1}{N} \log \mathbb{P}\left(M^{(N)}>N a\right)=a-\varrho^{+}-a \log \frac{a}{\varrho^{+}} .
$$

For $a \leq a^{-}:=\varrho^{-}$,

$$
\lim _{N \rightarrow \infty} \frac{1}{N} \log \mathbb{P}\left(M^{(N)}<N a\right)=a-\varrho^{-}-a \log \frac{a}{\varrho^{-}} .
$$

Example 1 Consider, with $d=2$, the scenario $\lambda_{1}=2, \mu_{1}=3, \lambda_{2}=\mu_{2}=1$; as a consequence $\varrho_{1}=\frac{2}{3}$ and $\varrho_{2}=1$, such that $i^{+}=2$. It is readily verified that $k=2$ (such that $i_{1}=1$ and $i_{2}=2$ ), whereas $I_{1}=\frac{1}{2}$ and $I_{2}=1$.

Using Theorem 1 , we find that the 'maximizing path' $f^{+}$is in state 1 until $t_{1}^{+}$, and state 2 thereafter, where $t_{1}^{+}$is given by

$$
t_{1}^{+}=\frac{1}{3} \log \left(\frac{\frac{2}{3}-0}{\frac{2}{3}-\frac{1}{2}}\right)=\frac{2}{3} \log 2 .
$$


As a consequence,

$$
C^{+}(t)=\kappa\left(f^{+}\right)= \begin{cases}\frac{2}{3}\left(1-e^{-3 t}\right), & t \in\left[0, t_{1}^{+}\right), \\ \frac{1}{2} e^{-\left(t-t_{1}^{+}\right)}+\left(1-e^{-\left(t-t_{1}^{+}\right)}\right), t \in\left[t_{1}^{+}, \infty\right)\end{cases}
$$

the expression for $t \geq t_{1}^{+}$simplifies to $1-\frac{1}{2} \sqrt[3]{4} e^{-t}$. Observe that, in agreement with our results, $C^{+}(t) \uparrow \varrho_{i^{+}}=\varrho_{2}$ as $t \rightarrow \infty$. It is easily verified that $C^{+}(t)$ is continuous in $t=t_{1}^{+}$.

The corresponding 'minimizing path' can be found analogously; it turns out that $f^{-}$is in state 2 until time $\log 2$, and in state 1 thereafter. It requires some elementary algebra to find that

$$
C^{-}(t):=\kappa\left(f^{-}\right)=\left\{\begin{array}{l}
1-e^{-t}, \quad t \in[0, \log 2) \\
\frac{2}{3}-\frac{4}{3} e^{-3 t}, t \in[\log 2, \infty)
\end{array}\right.
$$

observe that $C^{-}(t) \uparrow \varrho_{1}$ as $t \rightarrow \infty$, as expected.

The corresponding large deviations limit now immediately follows from Theorem 2.

\section{Fast timescale regime}

In this section, the process $M^{(N)}(t)$ results from scaling $\lambda$, as before, by a factor $N$, but now also the background process $J$ is sped up. The crucial idea is that $J$ is scaled by a factor $N^{1+\varepsilon}$, for some $\varepsilon>0$, and hence jumps at a faster time scale than the arrival process. The key finding of this section is that in this regime, as $N$ tends to $\infty$, the tail asymptotics of $M^{(N)}(t)$ increasingly behave as those of an $\mathrm{M} / \mathrm{M} / \infty$ queue with arrival rate $N \lambda_{\infty}$ and service rate $\mu_{\infty}$, where the definitions on $\lambda_{\infty}$ and $\mu_{\infty}$ are given in (3). The results and the proofs in this section are similar to those in [3], except of course for the approximation of the Poisson parameter of the scaled background process.

We denote the $N$-scaled background process by $\left(J^{\left(N^{1+\varepsilon}\right)}(t)\right)_{t \in \mathbb{R}}$. Let $\boldsymbol{L}^{\left(N^{1+\varepsilon}\right)}\left(t_{1}, t_{2}\right)$ be the empirical distribution of the background process in $\left[t_{1}, t_{2}\right)\left(\right.$ with $\left.t_{1}<t_{2}\right)$; its $i$-th component is the fraction of time spent in state $i$, for $i=1, \ldots, d$ (where obviously the $d$ components are non-negative and sum to 1 ), that is

$$
L_{i}^{\left(N^{1+\varepsilon}\right)}\left(t_{1}, t_{2}\right):=\frac{1}{t_{2}-t_{1}} \int_{t_{1}}^{t_{2}} 1\left\{J^{\left(N^{1+\varepsilon}\right)}(s)=i\right\} \mathrm{d} s .
$$


By $\boldsymbol{L}\left(t_{1}, t_{2}\right)$ we denote the counterpart of $\boldsymbol{L}^{\left(N^{1+\varepsilon}\right)}\left(t_{1}, t_{2}\right)$ for the non-scaled background process, where it is observed that, by changing the timescale,

$$
\boldsymbol{L}^{\left(N^{1+\varepsilon}\right)}\left(t_{1}, t_{2}\right) \stackrel{\mathrm{d}}{=} \boldsymbol{L}\left(N^{1+\varepsilon} t_{1}, N^{1+\varepsilon} t_{2}\right)
$$

It is well known that the following law of large numbers applies: for any $\mathscr{S} \subset \mathbb{R}_{+}^{d}$ such that $\pi$ is contained in the interior of $\mathscr{S}$, it holds that $\mathbb{P}(\boldsymbol{L}(0, t) \in \mathscr{S}) \rightarrow 1$ as $t \rightarrow \infty$; see standard textbooks on Markov chains, such as [11]. It is also a standard result (e.g. [7, Theorem 3.1.6]) that $\boldsymbol{L}(0, t)$ satisfies a large deviations principle with rate function

$$
\mathbb{I}(\boldsymbol{x}):=\sup _{\boldsymbol{u}>\mathbf{0}}\left(-\sum_{i=1}^{d} x_{i} \log \frac{\sum_{j=1}^{d} q_{i j} u_{j}}{u_{i}}\right) ;
$$

this function is positive except when $\boldsymbol{x}=\boldsymbol{\pi}$. Under mild regularity conditions imposed on the set $\mathscr{S}$, this large-deviations principle means that

$$
\lim _{t \rightarrow \infty} \frac{1}{t} \log \mathbb{P}(L(0, t) \in \mathscr{S})=-\inf _{\boldsymbol{x} \in \mathscr{S}} \mathbb{I}(\boldsymbol{x}) .
$$

Considering the case that $\mathscr{S}$ does not contain $\pi$, then the immediate consequence of this result is that the probability $\mathbb{P}(L(0, t) \in \mathscr{S})$ decays essentially exponentially.

In the sequel, we use the notation

$$
\varrho(t):=\frac{\lambda_{\infty}}{\mu_{\infty}}\left(1-e^{-\mu_{\infty} t}\right) .
$$

As in the previous section, we wish to characterize the probability that $M^{(N)}(t)$ exceeds $N a$, given that the system starts off empty. It is known that $N^{-1} M^{(N)}(t) \rightarrow \varrho(t)$, a.s. for $N \rightarrow \infty$; see [4, Lemma 3]. In this paper, we are concerned with the rare event that the number of jobs exceeds a level $N a$, with $a \geq \varrho(t)$. The following theorem states that the corresponding large deviations are those of Poisson random variables with parameter $\varrho(t)$.

Theorem 3 For $a \geq \varrho(t)$,

$$
\lim _{N \rightarrow \infty} \frac{1}{N} \log \mathbb{P}\left(M^{(N)}(t) \geq N a\right)=-\varrho(t)+a+a \log \frac{\varrho(t)}{a} .
$$

Proof Our starting point is again

$$
\mathbb{P}\left(M^{(N)}(t) \geq N a\right)=\mathbb{P}\left(P^{(N)}\left(\kappa\left(J^{\left(N^{1+\varepsilon}\right)}\right)\right) \geq N a\right) .
$$

For $\delta>0$, we define $\Delta(\pi)$ as a hypercube (of 'radius' $\delta$ ) around $\pi$ :

$$
\Delta(\pi):=\left(\pi_{1}-\delta, \pi_{1}+\delta\right) \times \cdots \times\left(\pi_{d}-\delta, \pi_{d}+\delta\right) .
$$


Also introduce, for $\zeta>0$, the event

$$
\mathscr{E}_{\delta}(\zeta, N):=\left\{\boldsymbol{L}^{\left(N^{1+\varepsilon}\right)}\left(0, \frac{t}{N^{\zeta}}\right) \in \Delta(\boldsymbol{\pi}), \ldots, \boldsymbol{L}^{\left(N^{1+\varepsilon}\right)}\left(\frac{\left\lceil N^{\zeta}\right\rceil-1}{N^{\zeta}} t, t\right) \in \Delta(\boldsymbol{\pi})\right\}
$$

Lower bound. Intersecting the event of our interest with a second event evidently leads to a lower bound. Following this idea, we determine the decay rate of the obvious lower bound

$$
\mathbb{P}\left(\left\{P^{(N)}\left(\kappa\left(J^{\left(N^{1+\varepsilon}\right)}\right)\right) \geq N a\right\} \cap \mathscr{E}_{\delta}\left(\frac{1}{2}, N\right)\right)
$$

The idea behind considering this intersection, is that we focus on the scenario that the empirical distribution of the background process is during $[0, t]$ in $\Delta(\pi)$, and hence systematically close to $\pi$.

To this end, first realize that, for any $\xi \in(0,1)$ and $N$ sufficiently large, by virtue of the law of large numbers for the empirical distribution of the background process; see for example [7, Theorem 3.1.6]:

$$
\begin{aligned}
\mathbb{P}\left(\mathscr{E}_{\delta}\left(\frac{1}{2}, N\right)\right) & \geq \prod_{i=1}^{\lceil\sqrt{N}\rceil} \min _{j_{i} \in\{1, \ldots, d\}} \mathbb{P}\left(\boldsymbol{L}\left(0, t N^{\frac{1}{2}+\varepsilon}\right) \in \Delta(\boldsymbol{\pi}) \mid J(0)=j_{i}\right) \\
& \geq(1-\xi)^{\lceil\sqrt{N}\rceil} .
\end{aligned}
$$

It is a direct consequence that

$$
\liminf _{N \rightarrow \infty} \frac{1}{N} \log \mathbb{P}\left(\mathscr{E} \delta\left(\frac{1}{2}, N\right)\right)=0
$$

We are thus left with determining a lower bound on the decay rate

$$
\liminf _{N \rightarrow \infty} \frac{1}{N} \log \mathbb{P}\left(P^{(N)}\left(\kappa\left(J^{\left(N^{1+\varepsilon}\right)}\right)\right) \geq N a \mid \mathscr{E}_{\delta}\left(\frac{1}{2}, N\right)\right)
$$

Now the crucial observation is that the Poisson random variable is stochastically increasing in its parameter. As a result, we need to find a lower bound on $N \kappa\left(J^{\left(N^{1+\varepsilon}\right)}\right)$, conditional on the event $\mathscr{E}_{\delta}\left(\frac{1}{2}, N\right)$. By picking in every segment and for every state (a) a lower bound on the state probability (still in $\Delta(\pi)$ ), as well as (b) the lower bound on the Poisson rate in this segment, it is readily verified that the following (deterministic!) lower bound applies:

$$
\varrho_{N}(t):=t \sqrt{N} \sum_{j=1}^{d} \sum_{i=1}^{\lfloor\sqrt{N}\rfloor}\left(\pi_{j}-\delta\right) \lambda_{j} \exp \left(-\frac{t}{\sqrt{N}} \sum_{\ell=1}^{d} \sum_{k=\lfloor 1+(s / t) \sqrt{N}\rfloor}^{\lceil\sqrt{N}\rceil}\left(\pi_{\ell}+\delta\right) \mu_{\ell}\right) .
$$


We thus obtain the lower bound

$$
\mathbb{P}\left(P^{(N)}\left(\kappa\left(J^{\left(N^{1+\varepsilon}\right)}\right)\right) \geq N a \mid \mathscr{E}_{\delta}\left(\frac{1}{2}, N\right)\right) \geq e^{-\varrho_{N}(t)} \frac{\left(\varrho_{N}(t)\right)^{\lceil N a\rceil}}{\lceil N a\rceil !} .
$$

Applying Stirling's factorial approximation, this leads to

$\liminf _{N \rightarrow \infty} \frac{1}{N} \log \left(e^{-\varrho_{N}(t)} \frac{\left(\varrho_{N}(t)\right)^{\lceil N a\rceil}}{\lceil N a\rceil !}\right) \geq \liminf _{N \rightarrow \infty} \frac{1}{N}\left(-\varrho_{N}(t)+N a+N a \log \frac{\varrho_{N}(t)}{N a}\right)$.

Define $\bar{\lambda}:=\sum_{i=1}^{d} \lambda_{i}$, and $\bar{\mu}:=\sum_{i=1}^{d} \mu_{i}$. From the expression for $\varrho_{N}(t)$ in (14), and realizing that (recognize a Riemann integral!)

$$
\frac{t}{\sqrt{N}} \sum_{\ell=1}^{d} \sum_{k=\lfloor 1+(s / t) \sqrt{N}\rfloor}^{\lceil\sqrt{N}\rceil}\left(\pi_{\ell}+\delta\right) \mu_{\ell} \rightarrow(t-s)\left(\mu_{\infty}+\delta \bar{\mu}\right)
$$

it is observed that, as $N \rightarrow \infty$,

$$
\frac{\varrho_{N}(t)}{N} \rightarrow \int_{0}^{t}\left(\lambda_{\infty}-\delta \bar{\lambda}\right) e^{-(t-s)\left(\mu_{\infty}+\delta \bar{\mu}\right)} \mathrm{d} s=\frac{\lambda_{\infty}-\delta \bar{\lambda}}{\mu_{\infty}+\delta \bar{\mu}}\left(1-e^{-t\left(\mu_{\infty}+\delta \bar{\mu}\right)}\right)=: \varrho^{(\delta)}(t)
$$

It follows that

$$
\begin{aligned}
\liminf _{N \rightarrow \infty} \frac{1}{N} \log \mathbb{P}\left(P^{(N)}\left(\kappa\left(J^{\left(N^{1+\varepsilon}\right)}\right)\right) \geq N a \mid \mathscr{E}_{\delta}\left(\frac{1}{2}, N\right)\right) \geq & -\varrho^{(\delta)}(t) \\
& +a+a \log \frac{\varrho^{(\delta)}(t)}{a} .
\end{aligned}
$$

The claimed lower bound follows by letting $\delta \downarrow 0$.

Upper bound. Again, we focus on scenarios in which the empirical distribution is consistently close to $\pi$. To this end, we consider the obvious upper bound

$$
\mathbb{P}\left(\left\{P^{(N)}\left(\kappa\left(J^{\left(N^{1+\varepsilon}\right)}\right)\right) \geq N a\right\} \cap \mathscr{E}_{\delta}\left(\frac{\varepsilon}{2}, N\right)\right)+\mathbb{P}\left(\mathscr{E}_{\delta}\left(\frac{\varepsilon}{2}, N\right)^{c}\right)
$$

Due to the union bound,

$$
\mathbb{P}\left(\mathscr{E}_{\delta}\left(\frac{\varepsilon}{2}, N\right)^{c}\right) \leq\left\lceil N^{\varepsilon / 2}\right\rceil\left(\max _{j \in\{1, \ldots, d\}} \mathbb{P}\left(\boldsymbol{L}\left(0, t N^{1+\frac{\varepsilon}{2}}\right) \notin \Delta(\boldsymbol{\pi}) \mid J(0)=j\right)\right) .
$$

The fact that $\pi$ is the unique minimizer of $\mathbb{I}(\cdot)$ entails that

$$
\lim _{N \rightarrow \infty} \frac{1}{N^{1+\frac{\varepsilon}{2}}} \log \mathbb{P}\left(\boldsymbol{L}\left(0, t N^{1+\frac{\varepsilon}{2}}\right) \notin \Delta(\boldsymbol{\pi}) \mid J(0)=j\right)=-\inf _{\boldsymbol{x} \notin \Delta(\boldsymbol{\pi})} \mathbb{I}(\boldsymbol{x})<0,
$$


and hence

$$
\limsup _{N \rightarrow \infty} \frac{1}{N} \log \mathbb{P}\left(\mathscr{E} \delta\left(\frac{\varepsilon}{2}, N\right)^{c}\right)=-\infty
$$

Using [7, Lemma 1.2.15], it now suffices to prove that

$$
\begin{aligned}
\limsup _{N \rightarrow \infty} \frac{1}{N} \log \mathbb{P}\left(P^{(N)}\left(\kappa\left(J^{\left(N^{1+\varepsilon}\right)}\right)\right) \geq N a \mid \mathscr{E}_{\delta}\left(\frac{\varepsilon}{2}, N\right)\right) \leq & -\bar{\varrho}^{(\delta)}(t) \\
& +a+a \log \frac{\bar{\varrho}^{(\delta)}(t)}{a},
\end{aligned}
$$

with $\bar{\varrho}^{(\delta)}(t)$ such that $\bar{\varrho}^{(\delta)}(t) \rightarrow \varrho(t)$ as $\delta \downarrow 0$. The remainder of the proof settles this issue.

To this end, we determine a (deterministic!) upper bound, conditional on $\mathscr{E}_{\delta}\left(\frac{\varepsilon}{2}, N\right)$, on the random variable $N \kappa\left(J^{\left(N^{1+\varepsilon}\right)}\right)$. Using a similar reasoning as in (14), it is readily verified that the following upper bound applies:

$$
\bar{\varrho}_{N}(t):=t N^{1-\frac{\varepsilon}{2}} \sum_{j=1}^{d} \sum_{i=1}^{\left\lceil N^{\frac{\varepsilon}{2}}\right\rceil}\left(\pi_{j}+\delta\right) \lambda_{j} \exp \left(-\frac{t}{N^{\frac{\varepsilon}{2}}} \sum_{\ell=1}^{d} \sum_{k=\left\lceil 1+(s / t) N^{\frac{\varepsilon}{2}}\right\rceil}^{\left\lfloor N^{\frac{\varepsilon}{2}}\right\rfloor}\left(\pi_{\ell}-\delta\right) \mu_{\ell}\right) .
$$

Chebycheff's inequality on the cumulant generating function of Poisson random variables [7, p. 30] gives

$$
\begin{aligned}
& \limsup _{N \rightarrow \infty} \frac{1}{N} \log \mathbb{P}\left(P^{(N)}\left(\kappa\left(J^{\left(N^{1+\varepsilon}\right)}\right)\right) \geq N a \mid \mathscr{E}_{\delta}\left(\frac{\varepsilon}{2}, N\right)\right) \\
& \leq \limsup _{N \rightarrow \infty} \frac{1}{N}\left(-\bar{\varrho}_{N}(t)+N a+N a \log \frac{\bar{\varrho}_{N}(t)}{N a}\right) .
\end{aligned}
$$

Pick $\delta$ sufficiently small that $\mu_{\infty}>\delta \bar{\mu}$. Combining the above findings, leads to the desired upper bound, realizing that, using the same reasoning as in the lower bound,

$$
\frac{\bar{\varrho}_{N}(t)}{N} \rightarrow \bar{\varrho}^{(\delta)}(t):=\frac{\lambda_{\infty}+\delta \bar{\lambda}}{\mu_{\infty}-\delta \bar{\mu}}\left(1-e^{-t\left(\mu_{\infty}-\delta \bar{\mu}\right)}\right)
$$

as $N \rightarrow \infty$; the claim follows immediately from $\bar{\varrho}^{(\delta)}(t) \rightarrow \varrho(t)$ as $\delta \downarrow 0$.

The following corollary follows from the the Gärtner-Ellis theorem [7, Theorem 2.3.6], in conjunction with the duality between the cumulant function and the large deviation rate function via the Legendre-Fenchel transform [7, Lemma 4.5.8].

Corollary 2 The limiting cumulant function of $M^{(N)}(t)$ corresponds to that of a Poisson random variable:

$$
\lim _{N \rightarrow \infty} \frac{1}{N} \log \mathbb{E} \exp \left(\vartheta M^{(N)}(t)\right)=\varrho(t)\left(e^{\vartheta}-1\right) .
$$


The above result directly carries over to the steady-state counterpart $M^{(N)}$ of $M^{(N)}(t)$. To this end, we define $\varrho:=\lim _{t \rightarrow \infty} \varrho(t)=\lambda_{\infty} / \mu_{\infty}$, and realize that $M^{(N)}$ has a Poisson distribution with mean

$$
N \int_{-\infty}^{0} \lambda_{J(s)} e^{-\int_{s}^{0} \mu_{J(r)} \mathrm{d} r} \mathrm{~d} s
$$

see for example [6]. Then the proof of the corollary below is essentially the same as the one for the transient case.

Corollary 3 For $a \geq \varrho$,

$$
\lim _{N \rightarrow \infty} \frac{1}{N} \log \mathbb{P}\left(M^{(N)} \geq N a\right)=-\varrho+a+a \log \frac{\varrho}{a}
$$

In addition, $N^{-1} \log \mathbb{E} \exp \left(\vartheta M^{(N)}\right) \rightarrow \varrho\left(e^{\vartheta}-1\right)$ as $N \rightarrow \infty$.

\section{Conclusions}

In the context studied in this paper, we have considered the model in which the departure rate depends on the current state of the background process; in other words, the hazard rate associated with the departure process may change in time. This is in contrast with the model analyzed in [2,3], where the jobs' service times are sampled upon arrival. It is noted that in the setting of $[2,3]$, the model could be extended to cover general service times, but observe that this variant does not have a natural counterpart in the hazard-rate case.

At a high level, both models have the same structure: (i) the number of jobs in the system obeys a Poisson distribution with random parameter, (ii) if the background process jumps slowly (relative to the job generation process), the large deviations resemble those of a Poisson random variable with a given mean, (iii) if the background process moves fast, the large deviations coincide with those of an ordinary infiniteserver queue. The novelty of the present paper lies predominantly in (ii) and (iii): the optimization program that yields the Poisson distribution for the slow timescale regime is intrinsically more complex than that of [2], while the proofs regarding the fast timescale regime are crucially different from those presented in [3], due to the more intricate way that the background process affects the Poisson parameter, as a consequence of the fact that the departure rate may change during the job's sojourn time.

The large-deviations results are in terms of logarithmic asymptotics. A topic for future research could relate to the identification of exact asymptotics, i.e., a function $f(\cdot)$ such that the ratio of the probability of interest and $f(N)$ tends to 1 as $N \rightarrow \infty$. In addition, the option of developing efficient rare-event simulation techniques could be further explored. 
Acknowledgments We thank Rami Atar (Technion) for useful discussions related to the proof of Theorem 1. The work of O. Kella is partially supported by Israel Science Foundation Grant No. 1462/13 and The Vigevani Chair in Statistics.

\section{Appendix}

Represent the elements of the set of combinations of arrival rates and service rates, i.e., $\left\{\left(\mu_{i}, \lambda_{i}\right) \mid i \in\{1, \ldots, d\}\right\}$, as points in the $(\mu, \lambda)$-plane. Also, we define, for any $i, j$ such that $\mu_{i} \neq \mu_{j}$,

$$
\gamma(i, j):=\frac{\lambda_{i}-\lambda_{j}}{\mu_{i}-\mu_{j}}
$$

denoting the slope between the points $\left(\mu_{i}, \lambda_{i}\right)$ and $\left(\mu_{j}, \lambda_{j}\right)$ in the $(\mu, \lambda)$-plane. Now consider the following algorithm in pseudocode to find $\varrho^{+}$, i.e., the maximum slope between the origin and any $\left(\mu_{i}, \lambda_{i}\right)$.

Algorithm 1 Input: $\lambda_{i}, \mu_{i}, i \in\{1, \ldots, d\}$ - Output: $\mathrm{k}, I_{0}, \ldots, I_{k}$ and $i_{1}, \ldots, i_{k}$.

0 . Set $\ell:=1, I_{0}:=0, \mathscr{A}_{0}:=\{1, \ldots, d\}$, and

$$
i_{1}:=\arg \min \left\{\mu_{i}: \lambda_{i}=\max _{j \in \mathscr{A}_{0}} \lambda_{j}\right\} .
$$

1. Let

$$
\mathscr{A}_{\ell}:=\left\{i: I_{\ell-1}<\gamma\left(i, i_{\ell}\right)<\varrho_{i_{\ell}} \text { and } \mu_{i}<\mu_{i_{\ell}}\right\}
$$

If $\mathscr{A}_{\ell}$ is empty go to step (3), otherwise, go to step (2). 2. Let

$$
I_{\ell}:=\min _{j \in A_{\ell}} \gamma\left(j, i_{\ell}\right), \quad i_{\ell+1}:=\arg \min _{i \in \mathscr{A}_{\ell}}\left\{\mu_{i}: \gamma\left(i, i_{\ell}\right)=I_{\ell}\right\}
$$

$$
\text { Set } \ell:=\ell+1 \text { and return to step (1). }
$$

3. Set $I_{\ell}:=\varrho_{i_{\ell}}$ and $S T O P$.

To aid in understanding, see Fig. 1 for an example with $d=7$ and $k=2$. Note that $i_{1}$ is the index of the node with the largest $\lambda_{i}$ and $i_{2}$ is that of the node with the largest $\varrho_{i}\left(=\varrho^{+}\right) . I_{0}$ is the slope zero, $I_{1}$ is the slope of the segment connecting $\left(\mu_{i_{1}}, \lambda_{i_{1}}\right)$ and $\left(\mu_{i_{2}}, \lambda_{i_{2}}\right)$, and $I_{2}=\varrho^{+}$. Note that in this case there are two indices $j$ for which $I_{1}=\gamma\left(i_{1}, j\right)$, and that $i_{2}$ is the one having the smaller value of $\mu_{j}$. Also note that there is a point (close to $(0,0)$ ) that if we connect a segment between it and $i_{2}$ the slope is greater than $I_{1}$. However, since this slope is also greater than $\varrho_{i_{2}}=\varrho^{+}$, the algorithm stops at $\ell=2$. The bold segments describe a concave function, which is the reason why $\varrho_{\ell}$, the slopes of the segment connecting $(0,0)$ and $\left(\mu_{i_{\ell}}, \lambda_{i_{\ell}}\right)$, increases in $\ell$ and in particular when the algorithm stops then $\varrho_{i_{\ell}}=\varrho^{+}$. Figure 2 demonstrates 
Fig. 1 Example with $d=7$ and $k=2$

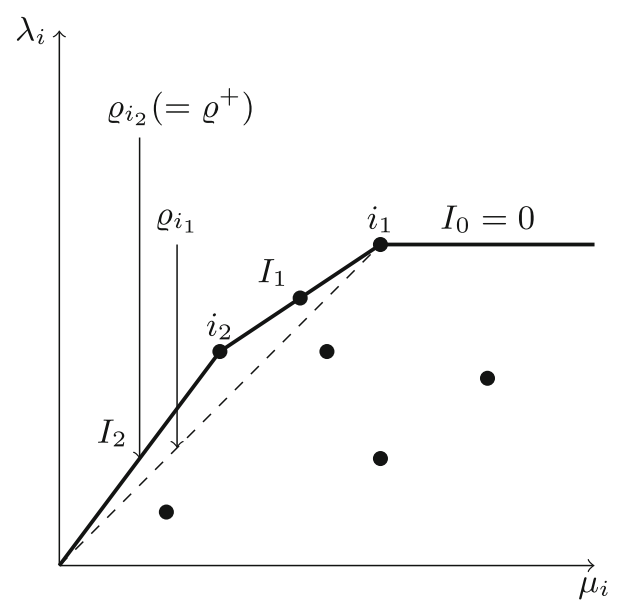

Fig. 2 Maximal value of $\lambda_{i}-c \mu_{i}$

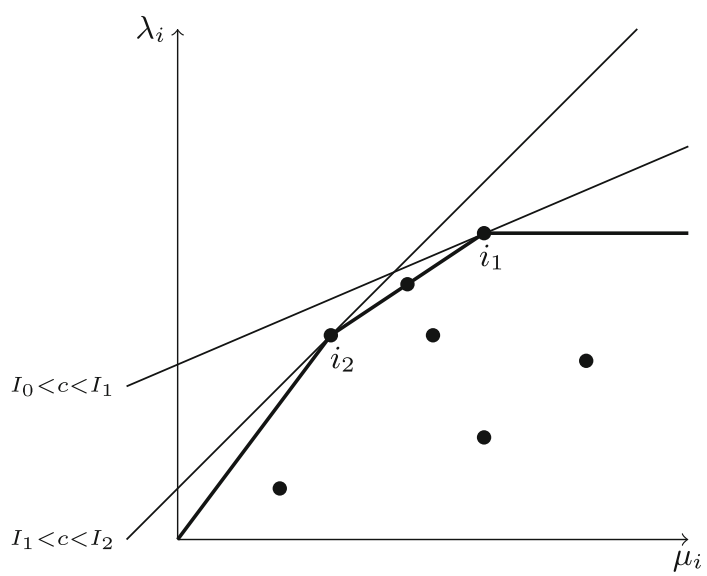

that the maximal value of $\lambda_{i}-c \mu_{i}$ is given by $i_{1}$ for $I_{0}<c<I_{1}$ and $\left(\mu_{i}, \lambda_{i}\right) \in$ $\left[\mu_{i_{2}}, \mu_{i_{1}}\right] \times\left[\lambda_{i_{2}}, \lambda_{i_{1}}\right]$ and by $i_{2}$ for $I_{1}<c<I_{2}$ and $\left(\mu_{i}, \lambda_{i}\right) \in\left[0, \mu_{i_{2}}\right] \times\left[0, \lambda_{i_{2}}\right]$.

Algorithm 2 Input: $\lambda_{i}, \mu_{i}, i \in\{1, \ldots, d\}$ - Output: $\mathrm{k}, I_{0}, \ldots, I_{k}$ and $i_{1}, \ldots, i_{k}$.

0 . Set $\ell:=1, I_{0}:=0, \mathscr{A}_{0}:=\{1, \ldots, d\}$, and

$$
i_{1}:=\arg \max \left\{\mu_{i}: \lambda_{i}=\min _{j \in \mathscr{A}_{0}} \lambda_{j}\right\} .
$$

1. Let

$$
\mathscr{A}_{\ell}:=\left\{i: I_{\ell-1}<\gamma\left(i, i_{\ell}\right)<\varrho_{i_{\ell}} \text { and } \mu_{i}>\mu_{i_{\ell}}\right\} .
$$

If $\mathscr{A}_{\ell}$ is empty go to step (3), otherwise, go to step (2). 
Fig. 3 Example with $d=7$ and $k=2$

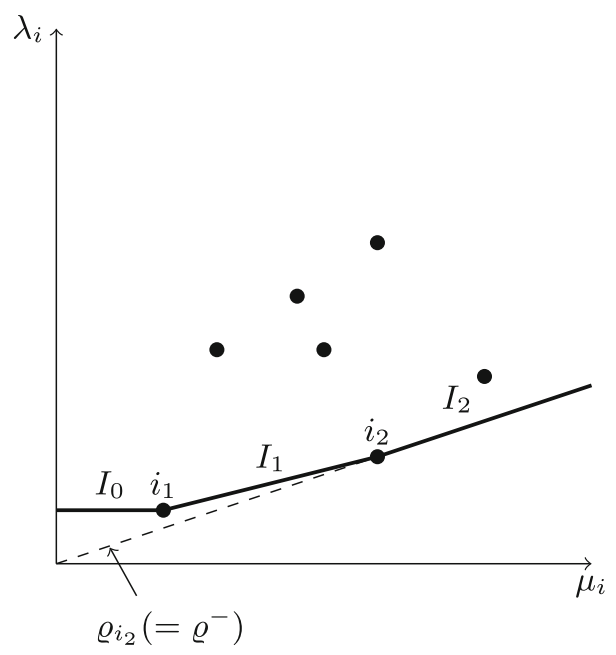

Fig. 4 Minimal value of $\lambda_{i}-c \mu_{i}$

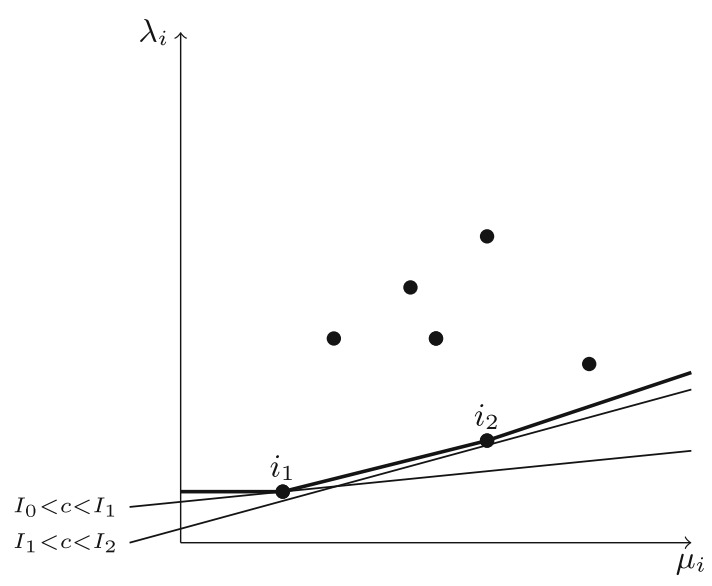

2. Let

$$
I_{\ell}:=\min _{j \in \mathscr{A}_{\ell}} \gamma\left(j, i_{\ell}\right), \quad i_{\ell+1}:=\arg \max _{i \in \mathscr{A}_{\ell}}\left\{\mu_{i}: \gamma\left(i, i_{\ell}\right)=I_{\ell}\right\}
$$

Set $\ell:=\ell+1$ and return to step (1).

3. Set $I_{\ell}:=\varrho_{i_{\ell}}$ and $\mathrm{STOP}$.

The corresponding figures are Figs. 3 and 4.

\section{References}

1. Blom, J., Mandjes, M.: A large-deviations analysis of Markov-modulated inifinite-server queues. Oper. Res. Lett. 41, 220-225 (2013) 
2. Blom, J., de Turck, K., Mandjes, M.: Rare-event analysis of Markov-modulated infinite-server queues: a Poisson limit. Stoch. Models 29, 463-474 (2013)

3. Blom, J., de Turck, K., Mandjes, M.: A central limit theorem for Markov-modulated infinite-server queues. In: Proceedings ASMTA 2013, Ghent, Belgium. Lecture Notes in Computer Science (LNCS) Series, vol. 7984, pp. 81-95 (2013)

4. Blom, J., Kella, O., Mandjes, M., Thorsdottir, H.: Markov-modulated infinite server queues with general service times. Queueing Syst. 76, 403-424 (2014)

5. Blom, J., Mandjes, M., Thorsdottir, H.: Time-scaling limits for Markov-modulated infinite-server queues. Stoch. Models 29, 112-127 (2013)

6. D'Auria, B.: M/M/ $\infty$ queues in semi-Markovian random environment. Queueing Syst. 58, 221-237 (2008)

7. Dembo, A., Zeitouni, O.: Large Deviations Techniques and Applications, 2nd edn. Springer, New York (1998)

8. de Turck, K., Mandjes, M.: Large deviations of an infinite-server system with a linearly scaled background process. Perform. Eval. 75-76, 36-49 (2014)

9. Fralix, B., Adan, I.: An infinite-server queue influenced by a semi-Markovian environment. Queueing Syst. 61, 65-84 (2009)

10. Keilson, J., Servi, L.: The matrix M/M/ $\infty$ system: retrial models and Markov modulated sources. Adv. Appl. Probab. 25, 453-471 (1993)

11. Norris, J.: Markov Chains. Cambridge University Press, Cambridge (1998)

12. O'Cinneide, C., Purdue, P.: The M/M/ $\infty$ queue in a random environment. J. Appl. Probab. 23, 175-184 (1986) 